\title{
Analysis of the Applicability of Design for Microassembly Theory to Biomedical Devices
}

\author{
Carsten Tietje ${ }^{1}$, Daniel Smale ${ }^{2}$, Steve Haley ${ }^{2}$, and Svetan Ratchev ${ }^{2}$ \\ ${ }^{1}$ Division of Engineering, The University of Nottingham Ningbo, China, \\ 199 Taikang East Road, Ningbo, 315100, China \\ carsten.tietje@nottingham.edu.cn \\ ${ }^{2}$ Precision Manufacturing Centre, The University of Nottingham, University Park, \\ Nottingham, NG7 2RD, UK \\ daniel.smale@nottingham.ac.uk, svetan.ratchev@nottingham.ac.uk
}

\begin{abstract}
This paper describes the application of design for microassembly $(\mathrm{D} \mu \mathrm{FA})$ theory to the designing and assembling of biomedical microdevices in order to cope with the market-specific requirements of the biomedical sector which can be seen as one of the most complex industrial areas for microassembly applications. It is shown how $\mathrm{D} \mu \mathrm{FA}$ can support the move from the research laboratory to industrial fabrication. The benefits of applying DF $\mu$ A theory to the development of a biomedical microdevice are clearly shown, i.e. savings in cost and time achieved in the early design stages. Therefore a practical case study containing a minifluidics blood separation device is introduced providing insight into the process of guiding the design process of microproducts.
\end{abstract}

\section{Introduction}

The development and manufacture of biomedical ${ }^{1}$ products for medical treatment represents a significant area in the European biomedical healthcare sector which is characterised by annual revenues in the order of 10 billion Euros [1, 2]. The development of biomedical devices is increasingly often accompanied by miniaturisation and functional integration and due to their intended area of application by complex environmental constraints.

The paper presented here describes the application of design for microassembly $(\mathrm{D} \mu \mathrm{FA})$ theory to the designing and assembling of such a biomedical microdevice in order to cope with the market-specific requirements. Furthermore, it is shown how D $\mu F A$ can support the move from the research laboratory to industrial fabrication which is often hindered by problems in selecting and implementing appropriate micromanufacturing processes [3-5]. The key to tackling these issues can be seen in the design stages: at present there is no sufficient link between microproduct design and production system design.

${ }^{1}$ The term biomedical is refers to biotechnology-derived medical devices and products that are mainly acquired for the medical sector (EMCC, 2007). 
The significant impact of the product design on the production and its cost has been analysed extensively [6-9]: it is easiest to make alterations early in the product development phases, consequently "[they are] the ideal and only time to get manufacturing cost right" [8].

\section{Definition of the Microassembly Case Study}

Assembly in the microdomain is more challenging than in the macrodomain because of different levels of maturity in the technology, differently occurring physical behaviour, and required microspecific processes. Although the differences have been elaborated on relatively extensively in the literature ${ }^{2}$ there has been a lack in developing appropriate DFA theories for the microdomain [17]. The case study presented here aims at showing the benefits of applying DF $\mu \mathrm{A}$ theory to microassembly in the biomedical sector. In addition, it serves the purpose of verifying the methodology. The practical test case is introduced in section 2.1 before its assembly requirements are defined in section 2.2.

\subsection{The Test Case - 3D Minifluidic Blood Separator}

The biomedical sector can be seen as one of the most complex industrial areas for microassembly applications due to the arising of additional requirements such as cleanliness, high reliability, biocompatibility, tight tolerances, and governmental regulations. Manufacturing for the healthcare market is also characterised by requirements such as traceability and documentation (e.g. compliance to Good Manufacturing Practices or rules imposed by the US Food and Drug Administration).

The product introduced here to verify the applicability of DF $\mu$ A theory is a minifluidics device consisting of several discs stacked on top of one another aiming at the separation of blood for analytic purposes. The product was developed as a demonstrator within the UK EPSRC grand challenge project 3D Mintegration (3DM). The dimensions of the product (particularly the channels in the discs) are calculated and designed to enable blood flow and plasma separation. ${ }^{3}$ Strong market relevance of the demonstrator is shown by fast growing rates of the healthcare sector worldwide, but particularly in the UK [18]. Therefore, establishment of cost-effectiveness is one of the main challenges imposing additional restrictions on the assembly process.

The joining process is one of the key processes in the manufacture of medical devices, this being due to the increasing sophistication of medical devices in terms of performance and therefore higher complexity of the devices' components. It is for this reason that the joining process has been singled out for this study as critical, imposing strict requirements (see section 2.2).

${ }^{2}$ On the differences between assembly in the macro- and in the microworld related to the required positional precision see [e.g. 10-13]. The changes in the physical behaviour such as scaling effects are analysed by [e.g. 14-16].

${ }^{3}$ Functioning is based on the flow behaviour of blood. The design is based on calculations and simulations carried out at the Universities Heriot-Watt, Greenwich, and Cambridge. The prototype parts were supplied by Cranfield University. 
Consequently the application $\mathrm{DF} \mu \mathrm{A}$ is focussed here on enabling the accurate joining of the parts demonstrating certain aspects of a methodology developed specifically for DF $\mu \mathrm{A}$ [19]. The following section described the parts and the joint requirements in more detail.

\subsection{Requirements Definition}

Five or more discs need to be joined on top of each other (in a stack). The discs, produced by micro-injection moulding, are $10 \mathrm{~mm}$ in diameter and $1 \mathrm{~mm}$ thick. The requirements with regard to the joining mechanism can be summarised as follows:

- Accuracy of joint and placement

The parts need to be accurately aligned in order to allow device functioning. Perpendicularity of features to the first surface is required. Alignment accuracy of \pm 20 micrometres is needed in $\mathrm{x}$ - and $\mathrm{y}$-directions, while rotational alignment is not necessary.

- Hermetic seal

To avoid contamination of the blood sample a hermetic seal between the layers is necessary. This is also the precondition for realising the blood flow.

- Contamination-free process

Contamination has to be avoided during the assembly. In addition, the parts' biocompatibility is not allowed to be effected.

- Low cost

Low production costs are crucial in order to compete in the healthcare market, which is moving from analysis in expensive central laboratories to costeffective point-of-care diagnostics.

- High volumes

The device is envisioned to be disposable and therefore requires extremely high volumes, creating a need for very short cycle times.

The case study has been chosen according to the above requirements to illustrate that the methodology can have an impact on the mass market or when up-scaling production. This addresses problems currently existing when transferring research prototypes to industrial practice (see section 1).

\section{Application of $\mathrm{DF} \mu \mathrm{A}$}

Figure 1 shows the development process from the initial design idea to the parts to be assembled. The three-dimensional product has been conceptually designed within the 3DM consortium and an embodiment design and prototype parts have then been provided. Accordingly the DF $\mu$ A methodology has been applied to existing parts. However, this offers advantages by allowing for testing the methodology's applicability and developing it further. As mentioned above, the key problem to be solved was how to join the parts while fulfilling the given requirements. The following subsections show how the process selection and the optimisation of the parts and joining process can be supported by the methodology. 

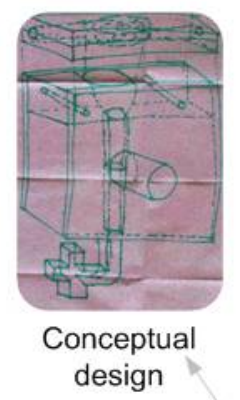
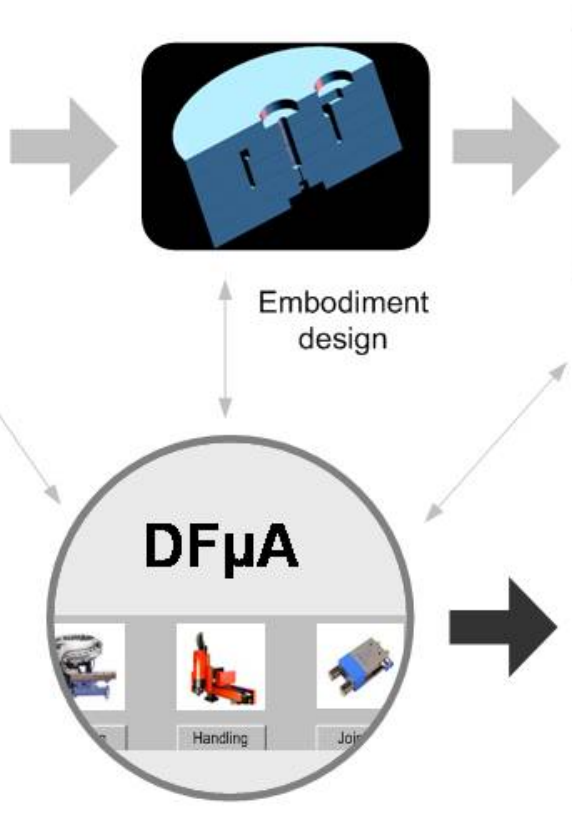
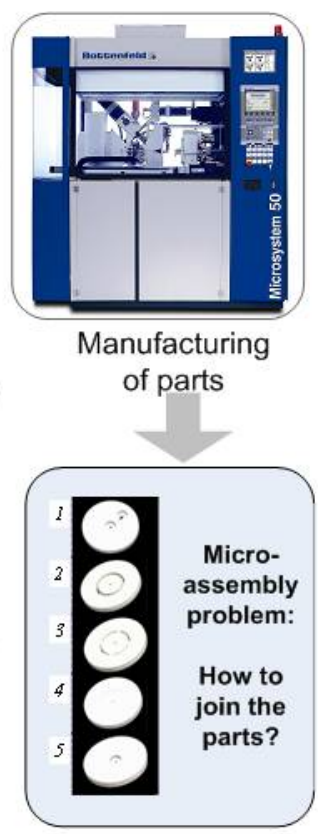

Fig. 1. Application of DF $\mu \mathrm{A}$ to the development process to the minifludics device

\subsection{Process Selection}

The assembly processes and equipment have been selected based on the defined requirements and the parts' characteristics (see section 2.2). Ultrasonic bonding was selected here particularly because of its cost-effectiveness and suitability to producing high volumes: the joining process can be automated to a very high degree with cycle times of below one second. In addition, ultrasonic bonding offers tight control with regard to dimensional tolerances while allowing for the realisation of very small joints on complex and fragile parts. Ultrasonic bonding can be optimised for use within a clean room environment, does not introduce contaminants or byproducts, and does not interfere with the biocompatibility of the parts to be joined. Finally, the ultrasonic bonding process provides the required hermetic seal without subjecting the parts to high temperatures which would lead to thermal deformations. It should be noted that the ultrasonic bonding process can produce small particulate matter which may affect operation within a cleanroom environment. It would therefore be necessary to monitor the process and potentially fit mitigative equipment, such as pumps or screening.

Figure 2 shows a screenshot of the software prototype that was developed to support the process selection. It illustrates the description of the ultrasonic bonding process which can be seen as an ideal assembly approach for applications within the medical sector. 


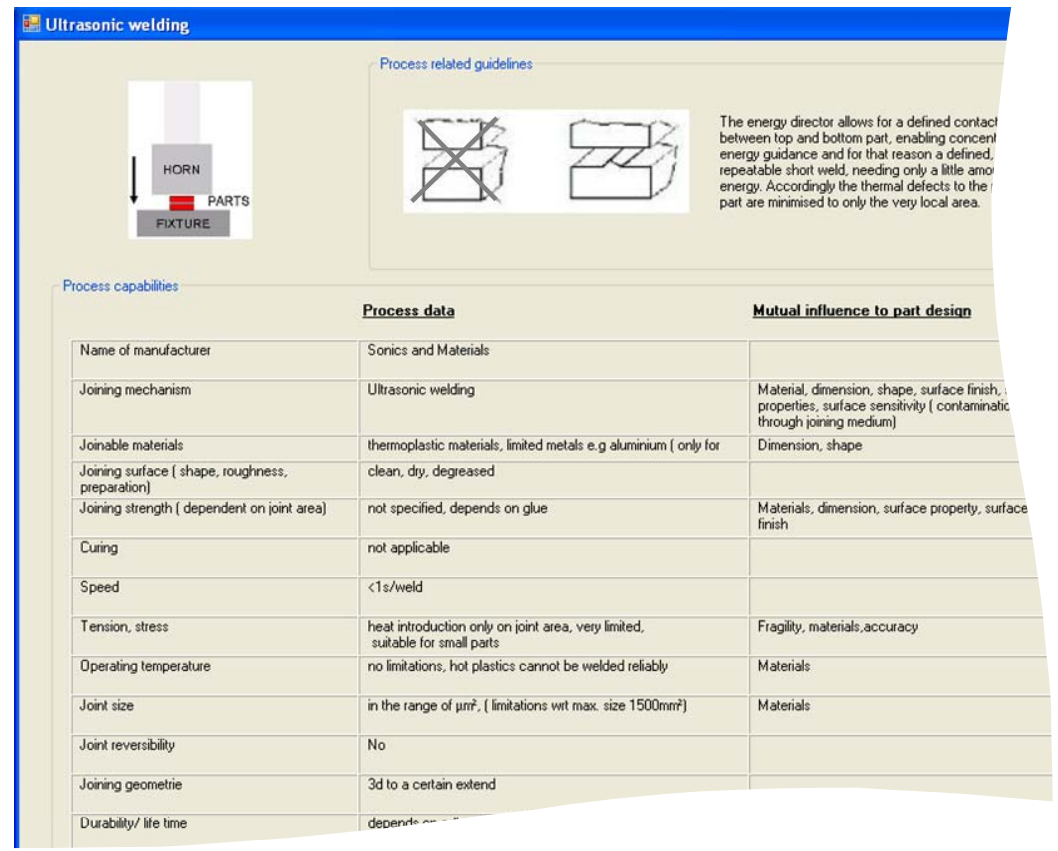

Fig. 2. Process sheet - Ultrasonic bonding (screenshot of the software prototype)

\subsection{Optimisation of Parts and Joining Process}

The optimisation and adaptation of the parts to be assembled and the selected joining process is a key step in the DF $\mu \mathrm{A}$ methodology. The assembly-oriented optimisation leads to parts that feature an area specifically designed to realising the joint (Figure 3). The parts contain an energy director that enables concentrated energy flow

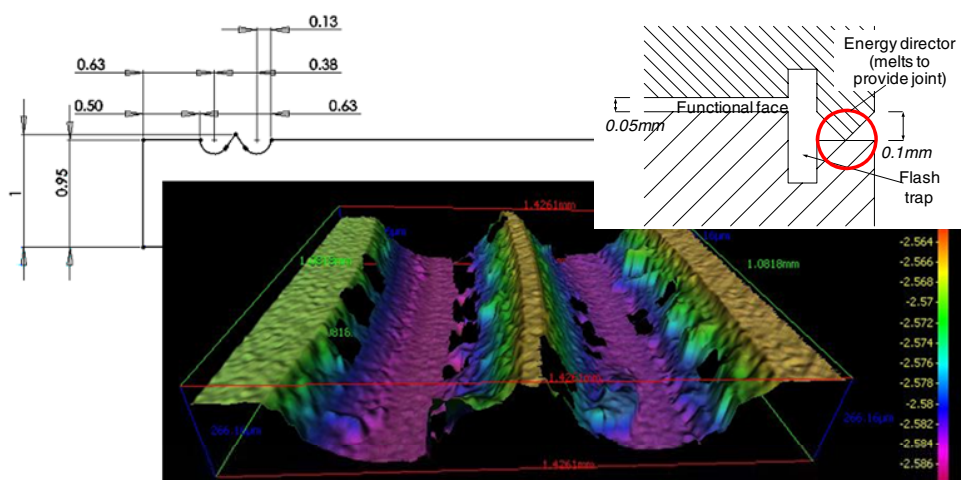

Fig. 3. Design adaptation for ultrasonic bonding - energy director 
resulting in a defined and repeatable short weld. In that way, the thermal defects to the plastic part are reduced to only a very local area. Trials showed that the surface roughness needs to be reduced to provide a defined contact surface between the top and bottom parts. Figure 3 shows the design of the part modification and a 3D picture including the surface roughness analysis. High alignment accuracy was realised through a fixture produced to hold the disks in the exact position required.

Figure 4 shows the results of these initial trials resulting in an unsatisfactory seal that cannot provide the desired device function of separating blood. The gap of 17-30 micrometres between two discs can be clearly identified in the SEM picture. Consequently the moulded parts were modified according to the guidelines related to the ultrasonic welding process (Figure 2). This optimisation of the joint resulted in a proper seal (see Figure 5).

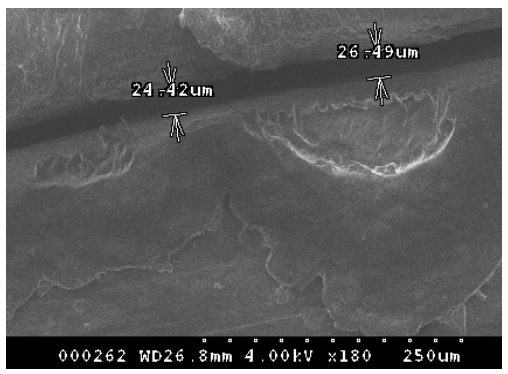

Fig. 4. SEM analysis - no sealed joint

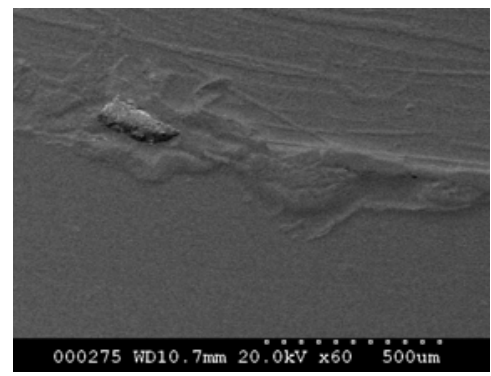

Fig. 5. Assembled device - hermetic joint

\section{Conclusion and Outlook}

The paper presented illustrates the benefits of the application of DF $\mu \mathrm{A}$ theory to the development of a biomedical microdevice. Therefore a practical case study was introduced providing insight into the process of guiding the design process of microproducts. A focus was laid on the support of selecting a joining process that meets the rigour requirements imposed by the $3 \mathrm{D}$ minifluidics blood-separation device. It was shown how the product's design was adapted and optimised towards the selected microassembly (here, joining) process. In the case presented ultrasonic welding process was identified as most suitable.

Applying the DF $\mu \mathrm{A}$ methodology shows that improvements and savings in cost and time can be achieved in the early design stages: the conventional design approach resulted in parts that were unusable, due to an unsatisfactory joining process, and needed reworking to fulfil the requirements of the test case. Based on that reworking, the embodiment design was changed, which resulted in the need for new micromoulds for the microinjection process. These steps are time-consuming and costly. The considering of process capabilities and related guidelines early in the design by applying $\mathrm{DF} \mu \mathrm{A}$ on the other hand leads to a directly optimised product design. 


\section{Acknowledgments}

The presented work is part of the UK EPSRC "Grand Challenge" research project "3D-Mintegration", that aims at a paradigm shift in manufacturing by developing the technologies and strategic approaches required for the production of highlyintegrated, cost-effective and reliable multi-functional $3 D$ miniaturised/integrated devices.

\section{References}

1. OECD, A framework for biotechnology statistics, Organisation for Economic Cooperation and Development (2005),

http: / / www . oecd. org/dataoecd/5/48/34935605.pdf

2. EMCC, Trends and drivers of change in the biomedical healthcare sector in Europe: Mapping report. European Foundation for the Improvement of Living and Working Conditions European Monitoring Centre on Change (2007), http: // www. eurofound. europa. eu/pubdocs / $2007 / 28 /$ en /1/ef0728en.pdf

3. Popovic, G., et al.: Examples for the technology selection method, in Micro fabrication processes - FSRM training course. FSRM, XIV-1 - XIV-8 (2004)

4. Alting, L., et al.: Micro Engineering. Annals of the CIRP 52(2), 635-657 (2003)

5. Hesselbach, J., Raatz, A. (eds.): mikroPRO, Untersuchung zum internationalen Stand der Mikroproduktionstechnik. Essen, Vulkan (2002)

6. Eversheim, W., Schuh, G. (eds.): Integrierte Produkt- und Prozessgestaltung. Springer, Berlin (2005)

7. Boothroyd, G., Dewhurst, P., Knight, W.: Product design for manufacture and assembly, 2nd edn. Marcel Dekker, New York (2002)

8. Miles, B., Swift, K.G.: Design for manufacture and assembly. Manufacturing Engineer 77(5), 221-224 (1998)

9. Reichenwald, R., Conrat, J.I.: Vermeidung von Aenderungskosten durch Integrationsmassnahmen im Entwicklungsbereich. Technical University Munich, Munich (1993)

10. Yang, G., Gaines, J.A., Nelson, B.J.: A flexible experimental workcell for efficient and reliable wafer-level 3D microassembly. In: IEEE International conference on robotics and automation, Seoul, pp. 133-138 (2001)

11. Scheller, T.: Untersuchung zu automatisierten Montageprozessen hybrider mikrooptischer Systeme, in Fakultaet Maschinenbau. Technical University Ilmenau, Illmenau (2001)

12. Cecil, J., Vasquez, D., Powell, D.: Assembly and manipulation of micro devices-A state of the art survey. Robotics and Computer-Integrated Manufacturing 23, 580-588 (2007)

13. Tichem, M., Lang, D., Karpuschewski, B.: A classification scheme for quantitative analysis of micro-grip principles. Assembly Automation 24(1), 88-93 (2004)

14. Van Brussel, H., et al.: Assembly of Microsystems. Annals of the CIRP 49(2), 451-472 (2000)

15. Fearing, R.S.: Survey of Sticking Effects for Micro-Parts. In: IEEE International Conference for Robotics and Intelligent Systems, IROS 1995, Pittsburgh (1995)

16. Ando, Y., Ogawa, H., Ishikawa, Y.: Estimation of attractive force between approached surfaces. In: Second Int. Symp. on Micro Machine and Human Science, Nagoya, Japan (1991) 
17. Tietje, C., Ratchev, S.: Design for microassembly - capturing process characteristics. In: Dimov, S., Menz, W., Toshev, Y. (eds.) 4M2007 Conference on Multi-Material Micro Manufacture. CRC Press Borovets, Boca Raton (2007)

18. Ratchev, S., Hirani, H.: Synergetic process integration for efficient micro and nano manufacture - roadmapping stage 1 results. MicroSapient (2006),

http: / / www.microsapient.org

19. Tietje, C., Ratchev, S.: Design for Micro Assembly - A methodology for product design and process selection. In: IEEE International Symposium on Assembly and Manufacturing (ISAM). Omnipress, Ann Arbor (2007) 